\title{
en docentes
}

universitarios,

propuesta y validación de un modelo teórico e instrumental

\section{University Professors}

Teaching Styles, Proposal and Validation of a Theoretical and Instrumental Model

\section{Estilos de ensino de professores universitários, Proposta e validação de um modelo teórico e instrumental}

Diana Margarita Abello Camacho* Carolina Hernández Valbuena** Christian Hederich Martínez ${ }^{* * *}$

*Profesora de la Universidad Pedagógica Nacional -Bogotá, D.C-. Magister en Educación de la misma Universidad. Correo electrónico: dabello@pedagogica.edu.co

**Profesora de la Universidad Pedagógica Nacional -Bogotá, D.C-. Magister en Educación de la misma Universidad. Correo electrónico: chernandezv@pedagogica.edu.co

***Profesor titular de la Universidad Pedagógica Nacional -Bogotá, D.CDoctor en Psicología de la Universidad Autónoma de Barcelona. Correo electrónico: hederich@pedagogica.edu.co

El artículo es resultado del proyecto de investigación "Consolidación del modelo teórico e instrumental de estilo de enseñanza" financiado por el centro de investigaciones de la Universidad Pedagógica Nacional -CIUP-y desarrollado por el grupo Estilos Cognitivos, categoría B en Colciencias. 


\section{Introducción}

$\mathrm{L}$ a necesidad de formar educadores que tengan una alta capacidad reflexiva es una de las competencias claves para lograr los retos actuales de la educación. A este respecto, diversos autores resaltan la importancia de la reflexión constante y crítica. Se enuncia así la capacidad de aprender y mejorar la práctica docente a partir del análisis e interpretación de la propia experiencia para mejorar su práctica (Mir, 2001; Stenhouse, 1985; Schön, 1992; Monereo, 1991). Dicha capacidad permite a los maestros estar constantemente atentos a los comportamientos que tienen en el aula, las estrategias que emplean y su impacto sobre la formación de los estudiantes. La capacidad de autoreflexión del maestro es entonces un componente esencial para lograr el mejoramiento de los procesos pedagógicos y didácticos en el aula.

En este marco de reflexión se resalta la necesidad de que los docentes dispongan de herramientas y estrategias que les permitan mejorar sus procesos reflexivos. Asimismo, se requiere tener acceso a miradas externas que les faciliten la interrelación de sus propias percepciones, en aras de configurar una comprensión más cercana a la realidad de su accionar en el aula.

En consecuencia, tales herramientas y estrategias deben facilitar la caracterización del accionar del docente en el aula de forma clara y neutral, brindando así una base sólida para favorecer la reflexión de su práctica. Esta caracterización de las herramientas de autoreflexión se conoce en términos investigativos con el término estilo de enseñanza. Constructo sobre el que diferentes autores han planteado diversos modelos que cristalizan, de diferente forma, el mismo entramado conceptual y teórico (Bennett, 1979; Lozano, 2001; Grasha, 2002; Gallardo y Ferreras, 2005; Martín, 2005; Camargo y Hederich, 2007; Martínez 2009).

Frente a las diferentes propuestas de estilos de enseñanza, Bennett (1979) analiza diversos estudios al respecto, concluyendo que las tipologías conocidas hasta ese momento desconocen la existencia de múltiples estilos intermedios y, además, están basadas, grosso modo, en muestras poco representativas.

En una revisión más reciente sobre los modelos de clasificación de los estilos de enseñanza, Camargo y Hederich (2007) categorizan las diferentes propuestas desarrolladas desde dos tradiciones, que denominan "tradición psicológica" y "tradición pedagógica". La primera se centra principalmente en la correspondencia entre los estilos cognitivos y de aprendizaje de los estudiantes y sus docentes. Desde esta tradición, se plantean claros modelos teórico-explicativos de los estilos, como los propuestos por Evans (2004) y Saracho (2003). Estos modelos, plantean propuestas generales de aplicación a la educación, pero no generan con claridad investigaciones sobre una enseñanza diferencial. Para McIntyre, (citado por Camargo y Hederich, 2007), tal generalidad per"En mite concluir que aún no existen caracterizaciones convincentes sobre el estilo con el que enseñan los docentes.

En segundo lugar, la tradición pedagógica define los estilos de enseñanza a partir de la necesidad de cualificación docente. Es así como un gran porcentaje de las propuestas, más que caracterizar la acción del docente en el aula, buscan identificar sus concepciones y creencias frente a la labor educativa. Ejemplos de estas dimensiones son las propuestas por Callejas y Corredor (2002), Grasha (2002), y Martín y Kempa (1991), entre otras. Desde el punto de vista de Camargo y Hederich (2007), este tipo de propuestas generan, dificultades en la definición del concepto "estilo de enseñanza"; tanto así que la misma investigación ha mostrado la enorme distancia que existe entre lo que piensa el docente que hace en el aula y lo que realmente hace (Reyes, Salcedo y Perafán, 1999; Calvo, 2001, citados por Camargo y Hederich, 2007; Traver, Sales, Doménech y Moliner, 2005).

En consecuencia, la denominada "tradición psicológica" hace la transferencia del estilo cognitivo a las preferencias didácticas del docente y deja de lado el aporte de la formación pedagógica y didáctica del docente. Por su parte, la "tradición pedagógica" al definir un mejor estilo en relación con los demás, reduce la identificación del estilo de enseñanza a una manera de evaluar el desempeño docente. Surge así, la necesidad de generar una propuesta que supere las limitaciones de ambas tradiciones con respecto a los estilos de enseñanza que, adicionalmente, cumpla con las características propias de la noción de 'estilo'. En este sentido, tal y como lo expone Hederich (2007), los estilos deben ser claramente diferenciadores y 
relativamente estables; deben además, integrar diferentes dimensiones del sujeto y ser neutrales a la hora de caracterizar denominada 'tralos comportamientos del docente. dición psicológica' hace la

Por otra parte, Camargo y Hederich señalan la necesidad de generar investigaciones relacionadas con la elaboración de instrumentos que permitan la identificación del 'estilo de enseñanza' de una forma objetiva y rápida. En la actualidad, los instrumentos existentes están elaborados bajo el esquema de autoreporte, lo cual presenta una dificultad importante al ofrecer una imagen distorsionada del comportamiento del maestro. Esta imagen es el resultado de las deformaciones cognitivas propias de la autoimagen del sujeto junto con aquella que quisiera proyectar a sus observadores.

Los planteamientos de Cooley (1902, citado por Gelles y Levine,1997) permiten explicar este fenómeno. El autor define dos tipos de identidad: la identidad personal concebida como las ideas y percepciones sobre sí mismo y la identidad pública, entendida como aquello que se quiere mostrar a los demás, es decir, la versión idealizada de cómo el sujeto desea ser visto. La imagen de sí mismo surge entonces de tres elementos: a) el imaginario que tiene el sujeto sobre lo que los otros perciben de él; b) las creencias del sujeto sobre la manera en que los otros juzgan lo que perciben de él y, c) el sentir del sujeto en relación con las anteriores reacciones.

La dificultad anteriormente mencionada en relación con el autoreporte, muestra la necesidad de generar otro esquema de identificación, que permita la conjunción de visiones distintas a la del maestro. En consecuencia se propone la elaboración de un instrumento que permita la caracterización del estilo docente a partir de la información proveniente de sus estudiantes, quienes son los sujetos que mayor tiempo comparten con éste y los receptores directos de su actuar. Para ello, la media estadística de las respuestas del conjunto de los estudiantes permitirá al docente tener una percepción más válida de sus conductas en el aula. Este punto de vista de los estudiantes podrá ser analizado y comparado por el profesor, para lograr así una lectura más amplia que le permita realizar un adecuado proceso reflexivo sobre su accionar en el aula.
La tesis de maestría, diseño y validación del un modelo teórico e instrumental para la identificación de estilos de enseñanza realizada por Abello y Hernández (2010) recoge esta necesidad de proponer un instrumento que dé cuenta de los estilos de enseñanza a partir de una visión pedagógica, asumiendo las características propias del aula, el contexto educativo y la formación docente, así como una visión psicológica, que retoma la influencia de los procesos internos del sujeto en su estilo de enseñanza. En ella, se presenta un modelo teórico compuesto por tres dimensiones, las cuales en su conjunto caracterizan el estilo de enseñanza de los docentes universitarios.

Adicionalmente se presenta el diseño del Inventario de Estilos de Enseñanza (IEE v.3). Instrumento que operacionaliza el modelo antes mencionado y ofrece la posibilidad de que sea diligenciado por el docente y por sus estudiantes. El instrumento ofrece entonces una doble finalidad: por un lado, permite al docente identificar cuál es su estilo de enseñanza; y por el otro, le facilita la confrontación de sus propias percepciones con la de sus estudiantes de forma tal que logre profundizar en los procesos de autoconocimiento y reflexión de su labor docente.

Este artículo expone los desarrollos posteriores realizados por el grupo de investigación en estilos $\operatorname{cognitivos}^{1}$ en torno a lo planteado por Abello y Hernández (2010). El propósito es cualificar tanto el modelo teórico como el instrumento que lo operacionaliza a partir de la realización de análisis psicométricos más profundos sobre la base de los datos existentes.

A continuación se presenta el diseño de la investigación, el modelo teórico, la posterior operacionalización en un instrumento psicométrico y su análisis.

\section{Diseño}

Con el fin de caracterizar los estilos de enseñanza de los docentes universitarios a partir del diseño y validación de un modelo teórico e instrumental; se

1 Grupo inscrito en Colciencias "Estilos cognitivos" de la Universidad Pedagógica Nacional, liderado por el Doctor Christian Hederich y al cual pertenecen las autoras del modelo. 
planteó un diseño mixto con prevalencia cuantitativa (ver figura 1).

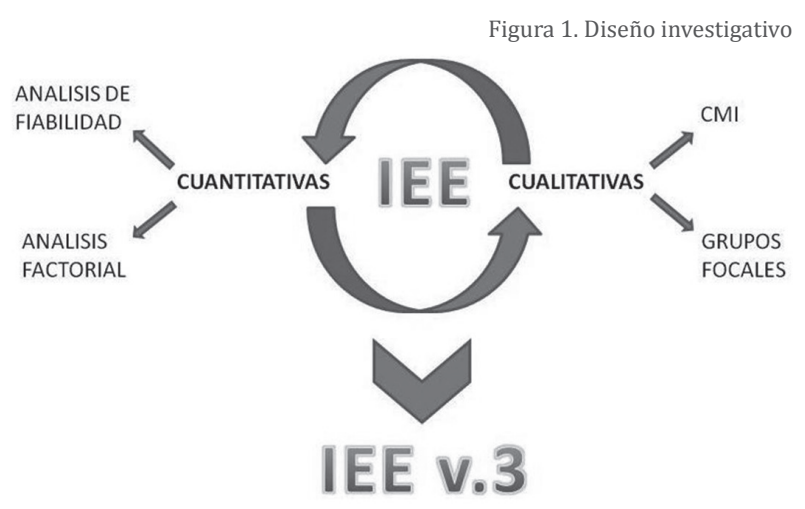

Dentro de las técnicas cualitativas empleadas se utilizó la Clasificación Múltiple de Ítems (CMI). Esta técnica tuvo el propósito de identificar los criterios que emplean los estudiantes para definir los estilos de enseñanza de sus docentes. Esta información se usó con el fin de apoyar la definición de las dimensiones del modelo propuesto. De igual forma se utilizaron los Grupos Focales, técnica que permitió la discusión y confrontación de las percepciones individuales de los estudiantes. Adicionalmente tal técnica facilitó la diferenciación de los puntos de vista colectivos e individuales. El objetivo de emplear esta técnica fue verificar la comprensión, neutralidad y estabilidad de los ítems del instrumento.

Las técnicas anteriormente descritas permitieron estructurar el Inventario de Estilos de Enseñanza -
IEE- en sus dimensiones constitutivas. Sobre estas dimensiones se procedió al diseño de ítems que, a través del análisis psicométrico clásico, se fueron depurando en sucesivas versiones. El resultado del proceso es el IEE en su tercera versión, a partir del cual se realizan las reflexiones del presente artículo.

Para el análisis de datos se utilizó el programa SPSS versión 15.0. En cuanto a la valoración psicométrica se realizaron los siguientes análisis:

Análisis de ítems: Se llevó a cabo mediante el índice de homogeneidad tanto en las dimensiones como en las subdimensiones. Se tienen en cuenta además las medidas descriptivas de frecuencia y varianza. Los ítems han sido analizados a partir del índice de homogeneidad (correlación elemento-total corregido). Para seleccionar los ítems se definió que debían tener una correlación ítem/factor superior a 0.30 .

Análisis de fiabilidad: Se utilizó el coeficiente de Alfa propuesto por Cronbach (1951), y corregido según la fórmula de Spearmann-Brown, para medir la consistencia interna del inventario completo y de cada una de las dimensiones y subdimensiones.

Validez de contenido: Se llevó a cabo principalmente a través de la recolección y el análisis de la información cualitativa proveniente de los estudiantes. Adicionalmente, los ítems del inventario se sometieron a la valoración de cinco docentes investigadores pertenecientes a la línea de estilos cognitivos de la Maestría en Educación de la Universidad Pedagógica Nacional, quienes evaluaron la redacción, el contenido y la clasificación de los ítems según las dimensiones.

Validez factorial: Se realizaron análisis factoriales exploratorios, utilizando el método de componentes principales con rotación varimax al conjunto de ítems de cada dimensión, explorando su estructura según la configuración de los factores específicos.

\section{Población y muestra}

La investigación en su totalidad se desarrolló con docentes y estudiantes de la Licenciatura en Educación con Énfasis en Educación Especial de la Universidad Pedagógica Nacional, sede Bogotá.

Para seleccionar la muestra se hizo un muestreo no probabilístico para el cual se tuvo en cuenta los siguientes criterios: en relación con la selección de los docentes se buscó igualar la muestra en cuanto al género y la formación inicial (licenciados y no licenciados), tal como lo visibiliza la Tabla 1.

Tabla 1. Muestra

\begin{tabular}{lllcc}
\hline & \multicolumn{2}{l}{ CRITERIO DE SELECCIÓN } & CANTIDAD & TOTAL \\
\hline \multirow{2}{*}{ DOCENTE } & \multirow{2}{*}{ Género } & Femenino & 14 & \\
\cline { 2 - 4 } & & Masculino & 10 & \multirow{2}{*}{24} \\
\cline { 2 - 4 } & $\begin{array}{l}\text { Formación } \\
\text { inicial }\end{array}$ & Licenciado & 12 & \\
\cline { 2 - 4 } ESTUDIANTES & No licenciado & N/A & 571 & 571 \\
\hline
\end{tabular}




\section{Modelo teórico para la comprensión del estilo de enseñanza}

La base epistemológica que permite comprender el estilo de enseñanza y las diferentes acciones $\mathrm{y}$ actores que se involucran en este proceso puede ser descrita desde la Teoría General de los Sistemas (TGS) planteada por Von Bertalanffy (1976). Los principios retomados de esta teoría ${ }^{2}$ y planteados desde el marco de la complejidad, permiten comprender de forma holística cómo se dan las interacciones de los actores implicados en el contexto del aula.

De igual forma, para comprender las interacciones dentro del sistema didáctico se retoma a Chevallard (1997) quien plantea las interrelaciones entre el docente, el estudiante y el saber como los elementos representativos de este sistema; haciendo evidente la interacción entre los protagonistas - el estudiante y el docente-, en relación directa con el objeto de conocimiento: el saber.

El modelo de estilo enseñanza propuesto, además de reconocer al docente y al estudiante, reconoce el grupo como un elemento adicional dentro del sistema didáctico. Esto en razón de que el grupo se comprende como un subsistema con características propias que, definido por el principio de totalidad de la TGS, resulta mayor a la suma de sus partes -estudiantes- mostrándose como un conjunto particular y claramente delimitado que genera interacciones diferentes dentro del aula.

Para el caso del subsistema saber el modelo retoma únicamente el saber enseñado definido por Chevallard (1997) cómo aquél que finalmente enseña el docente en la clase y que hace evidente la transposición didáctica del saber-sabio al saber-enseñado.

La interacción de estos elementos -docente, estudiante, grupo y saber-enseñado-, le da la particularidad al sistema. Por su parte el Inventario propuesto operacionaliza únicamente la identificación y caracterización de las conductas del docente que se llevan a cabo durante dicha interacción.

En la Figura 2 se estructuran las relaciones entre los elementos que definen el modelo a partir de una pirámide de base triangular, que en su cúspide ubica al docente y cada una de sus caras representa las dimensiones del estilo de enseñanza propuesto.

2 Los principios retomados de la teoría general de los sistemas son: 1) principio de totalidad; 2) principio de finalidad; 3) principio de retroalimentación; 4) principio de procesos; 5) principio de orden jerárquico y 6) principio de los elementos
Figura 2. Modelo de Estilo de Enseñanza
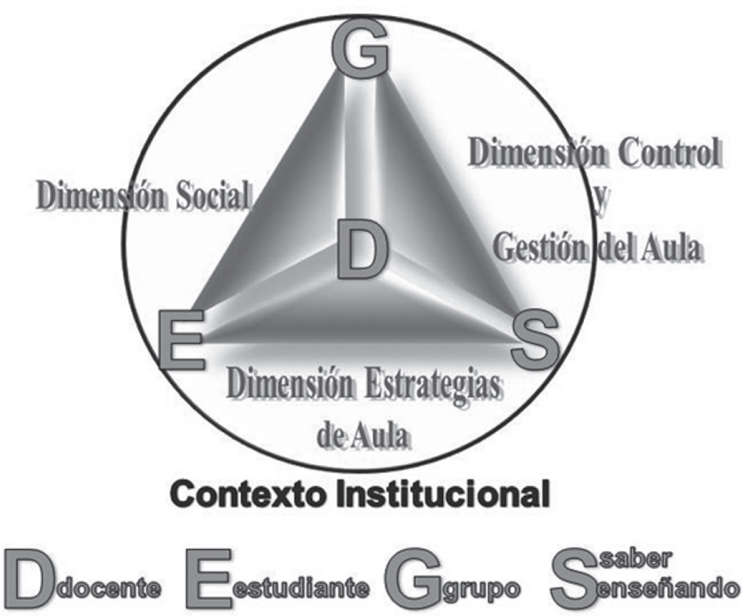

Es así como se generan las siguientes interacciones: a] docente-estudiante-grupo, de las cuales da cuenta la dimensión social; b] docente-grupo-saber enseñado, elementos que en su interacción definen la dimensión control y gestión del aula; y c] docenteestudiante-saber enseñado, que definen la dimensión estrategias de aula.

Esta descripción engloba lo que sucede en el aula de clases, ilustrado por la complejidad relacional que se da allí internamente y teniendo en cuenta que las interacciones entre los sujetos están mediadas, tanto por sus procesos internos, como por el contexto concretado en el marco institucional en el cual está inserta el aula.

\subsection{Dimensión social}

En esta dimensión se agrupan todos aquellos comportamientos que se dan en la interacción social entre el docente y el grupo o el docente y uno de los miembros del grupo. Se plantean dos subdimensiones: interacción docente - estudiante y toma de decisiones. La primera subdimensión parte de la percepción que tienen los estudiantes del docente en relación con el nivel de afectividad y cercanía emocional que se desarrolla en el aula. Para la construcción de esta subdimensión se tienen en cuenta los aportes realizados por Covarrubias y Piña (2004) sobre las representaciones sociales de los estudiantes y los desarrollos de Grasha (2002) con relación al tamaño y la distancia psicológica que perciben los estudiantes con el docente en el aula de clase.

Por su parte, la subdimensión toma de decisiones hace referencia a la interacción entre el docente y el grupo, mediada por el poder. La subdimensión se define así en términos del nivel en que el docente comparte el poder frente a diferentes acciones que se 


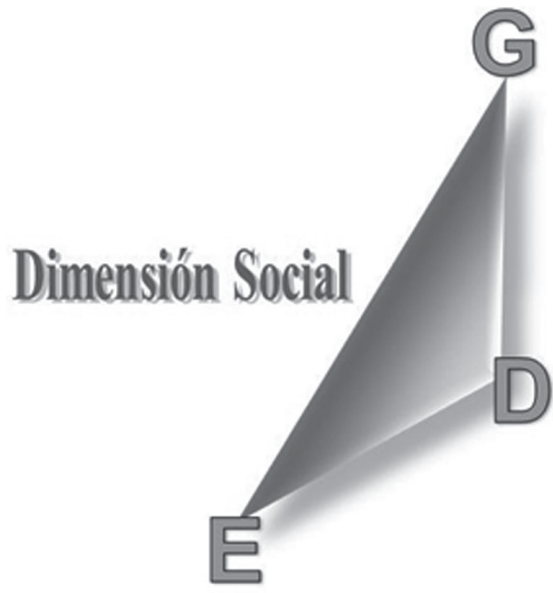

docente
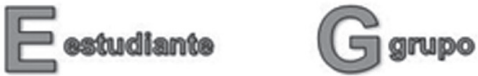

realizan en el marco de la clase. Para la construcción de esta subdimensión se retoman los planteamientos expuestos por la propuesta de Lewin, Lippit y White (1939; citados por Murillo, 2006) quienes proponen tres estilos de liderazgo con relación con el uso que el líder le da a la autoridad, sea éste de carácter autocrático, democrático o permisivo -laissez-faire-.

\subsection{Dimensión control y gestión del aula}

Esta dimensión está a su vez compuesta por dos subdimensiones. Por un lado, la interacción establecida entre el docente y el grupo mediada por las reglas de tipo explícito e implícito que caracterizan la subdimensión control del comportamiento. La otra subdimensión, estructuración de la enseñanza, se da a partir de la interacción entre el grupo y el saber-

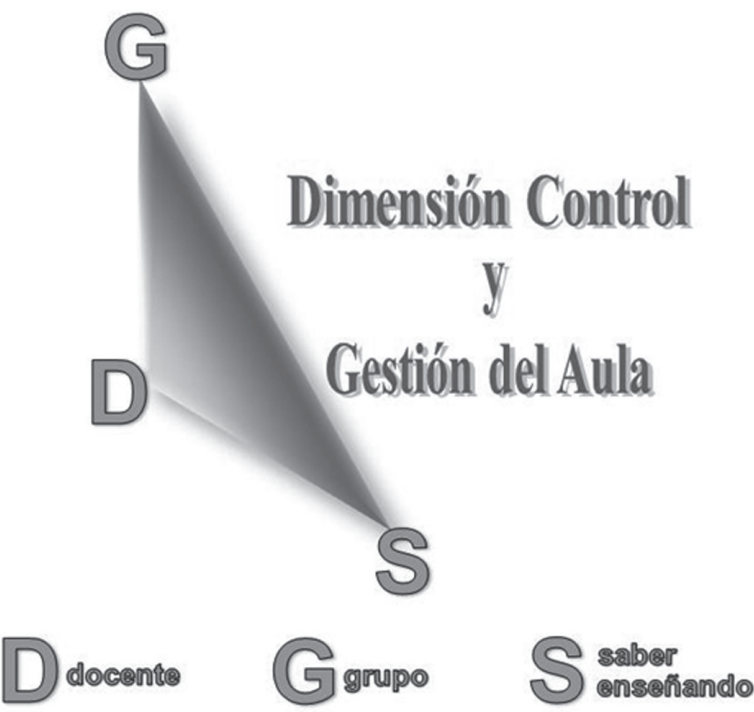

enseñado, mediada por la estructura que propone el docente para favorecer el aprendizaje.

Ambas subdimensiones parten de los preceptos de autoridad y de jerarquía en el aula planteados por Vázquez y Martínez (1996) quienes mencionan que el maestro es quien representa la autoridad dada por la institucionalidad. En consecuencia, tiene el poder de iniciar, mantener y terminar las interacciones en el aula así como de determinar su naturaleza.

Para el caso de la subdimensión control del comportamiento, ésta se centra en la importancia que el docente da a la instauración, seguimiento y control de las reglas normativas, que son aquellas relacionadas con el control conductual. Entre tanto, la subdimensión estructuración de la enseñanza hace referencia al nivel en el cual el docente explicita la estructura tanto de la sesión de clase, del curso completo, así como el grado de vigilancia y control que ejerce sobre el estudiante en relación con las actividades que debe desarrollar.

\subsection{Dimensión estrategias de aula}

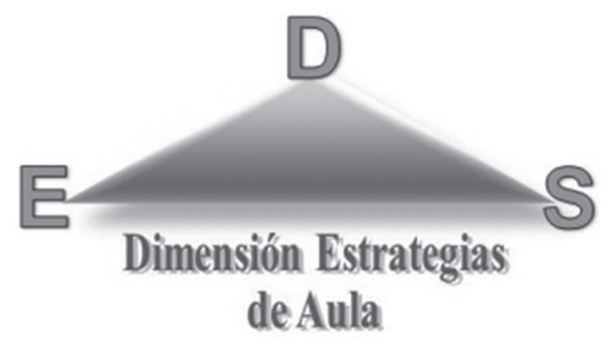

\section{D} docente
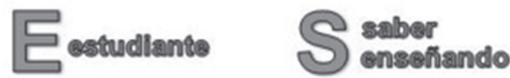

Aquello que caracteriza la dimensión estrategias de aula es la dinámica establecida entre el estudiante que aprende, el saber-enseñado y las acciones, y las estrategias y recursos que propone el docente para favorecer el proceso de aprendizaje.

Es una dimensión de carácter complejo, estructurada a partir de dos componentes:los elementos de la acción docente en el aula y los comportamientos de enseñanza según la propuesta de estilo de aprendizaje.

En el primer componente se asumen las acciones visibles durante el transcurso de la clase con relación a las estrategias, metodologías y recursos que plantea el docente. El segundo componente, que se define desde la propuesta estilística de Alonso, Gallego y Honey (1997), cuya perspectiva de estilos de aprendizaje pretende ser más detallada y basada en la acción propia de los sujetos, plantea entonces la definición de los 'estilos de aprendizaje' como la interiorización 

ciclo de aprendizaje ${ }^{3}$.

El dialogo entre estos dos componentes define cuatro tendencias (Cfr. Alonso, Gallego y Honey, 1997) en la forma en que el docente prioriza las estrategias de aula:

a. Tendencia activa: Hace referencia al nivel en el cual el docente prioriza la experiencia como estrategia didáctica. Caracteriza un docente que busca que el aprendizaje se genere desde la acción propia del estudiante. Promueve la exploración libre y el uso de las estrategias propias de cada estudiante y valora su capacidad de plantear propuestas y soluciones innovadoras.

b. Tendencia reflexiva: Esta tendencia hace referencia a los aspectos en los cuales el docente prioriza la reflexión como estrategia didáctica. Caracteriza a un docente que busca que el aprendizaje se genere a partir del análisis detallado y a profundidad de las situaciones o temáticas planteadas en la clase. Promueve la búsqueda de información y el diálogo constante con los estudiantes y valora su capacidad de argumentar y hacer reflexión crítica.

c. Tendencia teórica: Hace referencia al nivel en que el docente prioriza el estudio de teorías lógicas y complejas como estrategia didáctica. Caracteriza a un docente que busca que el aprendizaje se genere a partir de la revisión y análisis de modelos teóricos. que realiza el sujeto de una etapa determinada de su

Promueve la lectura analítica, la relación de conceptos a partir de herramientas de síntesis y valora los conocimientos adquiridos por los estudiantes y su capacidad de relacionarlos entre sí.

d. Tendencia pragmática: Hace referencia al nivel en que el docente prioriza el modelamiento como estrategia didáctica. Caracteriza a un docente que busca que el aprendizaje se genere a partir de la acción estructurada y guiada por el docente. Promueve el análisis de casos, analizando paso por paso los procedimientos adecuados y valora el desempeño de los estudiantes en la ejecución de procedimientos que den solución a situaciones prácticas.

Finalmente se proponen, como se muestra en la tabla 2, las dimensiones y sus respectivas subdimensiones, así como una definición breve para la estructuración del cuestionario.

En el presente trabajo se lleva a cabo una reflexión sobre las características psicométricas de las dos primeras dimensiones: dimensión social y dimensión control y gestión del aula, las cuales han resultado ser las más estables a lo largo del proceso de diseño e implementación del instrumento, arrojando altos niveles de confiabilidad. En un trabajo posterior se llevará a cabo el análisis de la dimensión estrategias de aula, la cual se configura como una dimensión compleja que requiere de un estudio particular.

Tabla 2. Dimensiones del estilo de enseñanza

\begin{tabular}{lll}
\hline \multicolumn{1}{c}{ DIMENSIÓN } & \multicolumn{1}{c}{ SUBDIMENSIONES } & \multicolumn{1}{c}{ CONCEPTUALIZACIÓN } \\
\hline \multirow{2}{*}{ Social } & $\begin{array}{l}\text { Interacción docente- } \\
\text { estudiantes }\end{array}$ & $\begin{array}{l}\text { Nivel de involucramiento emocional en la } \\
\text { interacción con el estudiante y con el grupo }\end{array}$ \\
\cline { 2 - 3 } Toma de decisiones & $\begin{array}{l}\text { Nivel en que el docente comparte el poder en la } \\
\text { toma de decisiones con el grupo }\end{array}$ \\
\hline \multirow{2}{*}{$\begin{array}{l}\text { Control y Gestión } \\
\text { del }\end{array}$} & $\begin{array}{l}\text { Estructuración de la } \\
\text { enseñanza }\end{array}$ & $\begin{array}{l}\text { Nivel en que el docente organiza y planifica los } \\
\text { procesos de enseñanza en el aula }\end{array}$ \\
\cline { 2 - 3 } Estrategias de & Control comportamiento & $\begin{array}{l}\text { Nivel en que el docente controla el } \\
\text { comportamiento del estudiante durante la clase }\end{array}$ \\
\cline { 2 - 3 } Aula & Tendencia activa & $\begin{array}{l}\text { Nivel en que el docente prioriza la experiencia } \\
\text { como estrategia didáctica }\end{array}$ \\
\cline { 2 - 3 } & Tendencia reflexiva & $\begin{array}{l}\text { Nivel en que el docente prioriza la reflexión como } \\
\text { estrategia didáctica }\end{array}$ \\
\cline { 2 - 3 } & Tendencia teórica & $\begin{array}{l}\text { Nivel en que el docente prioriza el estudio de } \\
\text { teorías lógicas y complejas como estrategia } \\
\text { didáctica }\end{array}$ \\
\cline { 2 - 3 } & Tendencia pragmática & $\begin{array}{l}\text { Nivel en que el docente prioriza el modelamiento } \\
\text { como estrategia didáctica }\end{array}$ \\
\hline
\end{tabular}

Los estilos de aprendizaje propuestos por Alonso, Gallego Honey (1997) son: el estilo activo, el estilo reflexivo, el estilo teórico y el estilo pragmático. 


\section{Inventario de estilos de enseñanza, versión 3 (IEE V3)}

El IEE v3 es un instrumento para la aplicación colectiva que cuenta con una versión para ser diligenciada por los estudiantes y una versión para ser diligenciada por el docente.

El Inventario recoge conductas referentes a las relaciones sociales que el docente establece, el nivel de control que ejerce y las estrategias que prioriza en el aula. Para el caso de este artículo se presentará la primera parte del instrumento que da cuenta de las dos dimensiones que serán analizadas. Su aplicación arroja resultados por cada una de las subdimensiones que estructuran a su vez las dimensiones propuestas por el modelo. La obtención de dichas puntuaciones se hace relevante en la medida en que el estilo del docente no es un constructo unitario y estático referido a los diferentes grupos humanos y temáticos a los cuales se enfrenta. De esa forma, el inventario da la posibilidad de reconocer las particularidades de la conducta del docente en el aula, así como las variaciones que éste realiza en relación con las particularidades del contexto.

En el Inventario, las conductas del docente aparecen descritas de forma precisa y específica, se describen situaciones concretas y no abstractas o hipotéticas, caracterizándose por ser neutrales y no valorativas para conservar la característica de neutralidad propia de los estilos.

Este Inventario cuenta con 27 ítems, distribuidos a través de las dos dimensiones propuestas para el análisis como lo muestra la Tabla 3.

Tabla 3. Distribución ítems IEE v3

\begin{tabular}{llcc}
\hline \multirow{2}{*}{ Dimensiones } & \multicolumn{1}{c}{ Subdimensiones } & $\begin{array}{c}\text { Cantidad } \\
\text { de items }\end{array}$ & $\begin{array}{c}\text { Número de } \\
\text { los items }\end{array}$ \\
\hline \multirow{2}{*}{ Social } & Interacción docente-estudiantes & 11 & 1 al 11 \\
\cline { 2 - 4 } & Toma de decisiones & 6 & 12 al 17 \\
\hline \multirow{2}{*}{ Controly Gestión del aula } & Estructuración de la enseñanza & 6 & 18 al 23 \\
\cline { 2 - 4 } & Control comportamiento & 4 & 24 al 27 \\
\hline
\end{tabular}

El inventario está organizado en una escala tipo Likert de cuatro opciones en la que se solicita al estudiante marcar con una X su nivel de acuerdo a partir de la siguiente escala: 1] total acuerdo; 2] moderado acuerdo; 3] moderado desacuerdo; 4] total desacuerdo. El siguiente es un ejemplo de la estructura.

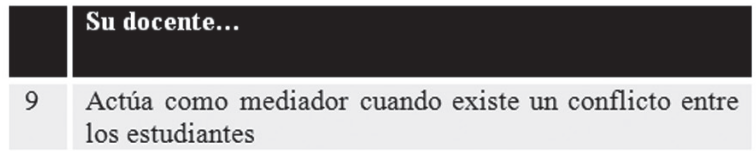

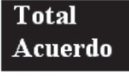

TA

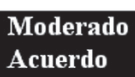

MA

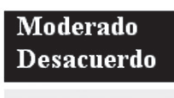

$\mathrm{MD}$
Total

Desacuerdo

TD

\section{Resultados}

Después de la aplicación del IEE v3 se obtienen los siguientes resultados:

\begin{tabular}{lllc}
\hline \multicolumn{1}{c}{ DIMENSIÓN } & ALFA & \multicolumn{1}{c}{ SUBDIMENSIÓN } & ALFA \\
\hline \multirow{2}{*}{ SOCIAL } & \multirow{2}{*}{0,962} & Interacción docente - estudiante & 0,967 \\
\cline { 3 - 4 } & & Toma de decisiones & 0,878 \\
\hline \multirow{2}{*}{ CONTROL Y GESTION DEL AULA } & \multirow{2}{*}{0,847} & Estructuración de la enseñanza & 0,832 \\
\cline { 3 - 4 } & & Control del comportamiento & 0,732
\end{tabular}

En general se observa que ambas dimensiones superan los mínimos establecidos, mostrando un instrumento con altos niveles de consistencia interna. A continuación se presenta el análisis detallado de cada dimensión:

\subsection{Dimensión social}

La dimensión está compuesta por 17 ítems y obtiene un alfa total de 0,962 . Por su parte el análisis factorial indica claramente la pertinencia de pre- sentar las dos subdimensiones, que en su conjunto explican el 75,281\% de la varianza:

La subdimensión interacción docente - estudiantes incluye 11 ítems y obtiene un alfa total de 0.967. Este valor se considera alto en relación con los estándares internacionales para la medición de la consistencia interna de los ítems. Los resultados del análisis factorial corrido sobre los 11 ítems indican la pertinencia de considerar un solo factor (por el criterio del autovalor mayor que 1) el cual explica el 75,933\% de la varianza. Esto muestra que existe consistencia interna en esta subdimensión. 
Se observa que en los ítems de esta subdimensión se obtienen bajos niveles de dispersión de los datos arrojando una dispersión de 0,83. Es posible entonces afirmar que los estudiantes tienen un alto nivel de acuerdo en relación con las conductas que emite su docente en la subdimensión social. A continuación se presentan los ítems de la subdimensión interacción docente - estudiantes

\begin{tabular}{l} 
Actúa como mediador cuando existe un conflicto entre los estudiantes \\
Los estudiantes pueden acercarse para compartir sus problemas personales \\
Identifica el ambiente emocional del grupo teniéndolo en cuenta para el desarrollo de la clase \\
\hline Manifiesta interés por el bienestar de los estudiantes más allá del contexto académico \\
\hline Muestra interés por conocer y vincularse con los estudiantes \\
Se relaciona con los estudiantes de manera cercana \\
\hline Reconoce las individualidades dentro del grupo \\
Genera un espacio de socialización antes de iniciar la clase \\
\hline Está pendiente de las situaciones personales de los estudiantes que pueden afectar su desempeño académico \\
\hline Saluda a los estudiantes fuera de la clase \\
\hline Se preocupa por el progreso individual de los estudiantes
\end{tabular}

La subdimensión toma de decisiones incluye 6 ítems y obtiene un alfa total de 0.878 . Este valor se considera alto en relación con los estándares internacionales para la medición de la consistencia interna de los ítems. Los resultados del análisis factorial corrido sobre los 6 ítems indican la pertinencia de considerar un solo factor (por el criterio del autovalor mayor que 1) el cual explica el 63,403\% de la varianza. Esto muestra que existe consistencia interna en esta subdimensión.

Se observa que en los ítems de la subdimensión se obtienen bajos niveles de dispersión de los datos arrojando una desviación estándar de 0,78. Es posible entonces afimar que, de igual manera para el caso de esta subdimensión, los estudiantes tienen un alto nivel de acuerdo en relación con las conductas que emite su docente. A continuación se presentan los ítems que componen esta subdimensión:

\begin{tabular}{l} 
Es flexible con las actividades propuestas en el programa \\
Prefiere llegar a acuerdos que imponer decisiones \\
\hline En consenso con los estudiantes se plantean las normas de la clase (hora de ingreso, receso, uso del celular) \\
\hline Frente a situaciones imprevistas, el docente acuerda con los estudiantes las acciones a seguir \\
\hline Adapta las temáticas de la clase a los intereses y necesidades particulares del grupo \\
\hline Escucha y toma en cuenta las razones del estudiante cuando se equivoca o falla \\
\hline
\end{tabular}

\subsection{Dimensión control y gestión del aula}

La dimensión está compuesta por 10 ítems y obtiene un alfa total de 0,847 . Por su parte el análisis factorial arroja tres factores de los cuales es pertinente considerar los dos primeros que explican el $51.4 \%$ de la varianza. Esta decisión se toma ya que el último factor, compuesto por el ítem realiza las evaluaciones planteadas en el programa, y el ítem es estricto con las fechas de entrega de trabajos, generan una nueva categoría que no muestra una relación directa con el modelo de estilo de enseñanza propuesto. Su ubicación se toma entonces a partir del análisis teórico y en concordancia con el segundo puntaje mayor expresado en los otros factores. Adicionalmente se encuentra que al realizar los análisis factoriales por subdimensiones estos se muestran coherentes en relación con las subdimensiones planteadas, mostrando además adecuados niveles de correlación.

Sobre esta base se exponen a continuación las dos subdimensiones:

La subdimensión estructuración de la enseñanza incluye 6 ítems y obtiene un alfa total de 0.841. Este valor se considera adecuado en relación con los estándares internacionales para la medición de la consistencia interna de los ítems. Adicionalmente no presenta ítems por debajo del mínimo establecido. Los resultados del análisis factorial corrido sobre los 6 ítems indican la pertinencia de considerar un único factor (por el criterio del autovalor mayor que 1) el cual explica el 56,979\% de la varianza.

Se observa que los ítems de esta subdimensión obtienen bajos niveles de dispersión en los datos, arrojando una desviación estándar de 0,73. Se muestra así que los estudiantes tienen un alto nivel de acuerdo en relación con las conductas que emite su docente en la subdimensión estructuración de la enseñanza.

A continuación se muestran los ítems que la componen:

Introduce la clase enunciando las actividades que se desarrollaran

Lleva a cabo rutinas claramente establecidas durante la sesión de clase Desarrolla la clase siguiendo una estructura clara

Retroalimenta el desempeño de los estudiantes a lo largo del semestre

Realiza las evaluaciones planteadas en el programa

Es puntual para comenzar la clase 
La subdimensión control del comportamiento incluye 4 ítems y obtiene un alfa total de 0.732 . Este valor se considera aceptable en relación con los estándares internacionales para la medición de la consistencia interna de los ítems. Los resultados del análisis factorial corrido sobre los 4 ítems indican la pertinencia de considerar un único factor el cual explica el 55,982\% de la varianza. Se observa que los ítems de la subdimensión obtienen bajos niveles de dispersión en los datos, con una desviación estándar de 0,79.

A continuación se muestran los ítems que componen la subdimensión:

Verifica que el estudiante ha realizado una preparación previa para la clase
Es estricto con las fechas de entrega de trabajos
Exige de sus estudiantes un "comportamiento adecuado" durante la clase
Recuerda constantemente las normas de la clase

\section{Discusión}

\subsection{Sobre el carácter estilístico de la propuesta}

A continuación se realiza el análisis de la estructura subyacente al instrumento a la luz de la noción de estilo propuesta por Hederich (2007).

La aplicación del instrumento a los 24 docentes participantes mostró que las diferentes dimensiones presentan un carácter altamente diferenciador. Para cada una de las dimensiones propuestas fue posible identificar docentes que se ubicaron en los dos extremos de la dimensión además de en su punto medio. Esta distribución de la muestra a lo largo de cada dimensión se cumple con la propiedad de diferenciar los comportamientos de los docentes incluso hasta el extremo, cuando se consideran las diferentes dimensiones en su conjunto ${ }^{4}$.

Asimismo, es posible observar la neutralidad de la propuesta en la "Las dimensiones operacionalización realizada de las características del docente en ítems centrados en conductas observables. La construcción de las dimensiones se estructuró de tal forma que no partiera del principio de considerar una u otra conducta como positiva o negativa, deseada o indeseada, sino que se originara en propuestas relacionadas en acciones no valorativas. Por otra parte, el hecho de que las puntuaciones finales se obtengan a partir de

4 Un análisis detallado de los resultados con los docentes de la muestra se presentará en un artículo posterior. la mirada de diversos estudiantes, permite lograr una mayor neutralidad frente a las percepciones valorativas que estos pudieran tener.

La propuesta es integradora en la medida en que se define el estilo a partir de la interacción de diversas dimensiones del ser humano. Las dimensiones propuestas abarcan ítems que diferencian las acciones propias de la disciplina pedagógica de las características psicológicas propias del docente. La dimensión estrategias de aula responde con mayor énfasis a la disciplina pedagógica. Por su parte la dimensión social enfatiza en las características psicológicas propias del docente. Finalmente la dimensión control y gestión del aula tiene un carácter pedagógico en la subdimensión estructuración del conocimiento y psicológico en la subdimensión control del comportamiento. Se propone así un modelo que brinda al docente una lectura abarcadora de su accionar en el aula, superando a otros que se centran únicamente en las estrategias pedagógicas.

Con relación a la estabilidad de los comportamientos que pretendemos describir, infortunadamente no es posible evidenciar con los procesos realizados hasta ahora, si el modelo cumple o no con estas características. Para ello es necesario, por un lado, realizar la aplicación del inventario a una muestra de mayor amplitud en la cual se analice la caracterización del docente en diferentes espacios académicos, y por otro lado, hacerlo a través de un diseño de medidas repetidas. psicológicas propias del docente."

\subsection{Sobre las características técnicas del IEE v.3.1}

En conclusión, se puede afirmar que se logra construir un instrumento consistente estadística- 
mente. A su vez los análisis factoriales muestran que se logra operacionalizar con claridad y coherencia dos -social y control y gestión del aula- de las tres dimensiones propuestas en el modelo, en la medida en que éstas se diferencian en componentes separados. Frente a la tercera dimensión, estrategias de aula, es necesario mejorar las cualidades psicométricas que se presentan, pues su complejidad dificulta la operacionalización.

Se resalta la calidad de los ítems diseñados con relación a su carácter conductual y su neutralidad, lo cual se evidencia en los niveles de coincidencias encontradas ente las percepciones del docente y las de los estudiantes. Lo anterior permite que, aquéllos que diligencian el cuestionario den cuenta en mayor proporción de sus acciones y no de sus creencias o prejuicios, con lo cual se supera las dificultades de los instrumentos de autoreporte. El instrumento planteado ofrece entonces una doble finalidad: Por un lado, permite al docente identificar cuál es su estilo de

\section{Bibliografía}

Abello, D.; Hernández, C. (2010), Diseño y validación de un Modelo teórico e instrumental para la identificación de estilos de enseñanza en docentes universitarios, [tesis de Maestría], Bogotá, Universidad Pedagógica Nacional, Maestría en Educación.

Alonso, C.; Gallego, D.; Honey, P. (1997), Los estilos de aprendizaje, Bilbao, Ediciones mensajero.

Bertalanffy, L. (1976), Teoría General de Sistemas, Petrópolis, Vozes.

Bennett, N. (1979), Estilos de enseñanza y progreso de los alumnos, Madrid, Morata.

Camargo, A.; Hederich, C. (2007), El estilo de enseñanza: un concepto en búsqueda de precisión, en: Pedagogía y Saberes, Universidad Pedagógica Nacional, Facultad de educación, núm.26, pp 31- 40.

Callejas, M., corredor, M. (2002), La renovación de los estilos pedagógicos: colectivos para la investigación y la acción en la universidad, en: Revista docencia universitaria, Universidad Industrial de Santander, vol.3, núm.1.

Covarrubias, P. (2004), La interacción maestro-alumno y su relación con el aprendizaje, en: Revista latinoamericana de estudios educativos, 1er. Trimestre, núm. 1, pp. 47-84

Chevallard, Y. (1997), La transposición didáctica. Del saber sabio al saber enseñado, Buenos Aires, AIQUE. enseñanza y, por el otro, le facilita la confrontación de sus propias percepciones con la de sus estudiantes de tal forma que se logre profundizar en los procesos de autoconocimiento y reflexión de su labor docente.

Finalmente, seha de señalar que la investigación realizada genera como producto el IEE v3. Este Inventario se traduce en un instrumento altamente confiable para la caracterización del estilo de enseñanza del docente en un espacio académico particular. El instrumento, por su parte, abre las puertas a futuras investigaciones y discusiones sobre los Estilos de enseñanza y su relación con distintas variables propias del docente y del estudiante que abarcan tanto las características personales, como las educativas y la influencia del contexto institucional. Este trabajo está siendo desarrollado en la actualidad, en el marco del proyecto "Consolidación del modelo teóricoinstrumental del estilo de enseñanza", que se adelanta en el Centro de Investigaciones de la Universidad Pedagógica Nacional.

Evans, C. (2004), Exploring the relationships between cognitive style and teaching style, en: Educational psychology, vol.24, pp. 509-530

Gallardo, B., Ferreras, A. (2005), "Perfiles de docencia y evaluación de los profesores universitarios valencianos", [Adenda a la ponencia] "El cambio en la cultura docente en la universidad ante el espacio europeo de educación superior", XXIV seminario interuniversitario de la teoría de la educación, Valencia.

Gelles, R. \& Levine, A. (1997), Introducción a la sociología con aplicaciones a los países de habla hispana. 5a. ed., México, McGraw-Hill.

Grasha, A. (2002), Teaching with Style, San Bernandino CA, Alliance Publishers.

Hederich, C. (2007), Estilo cognitivo en la dimensión dependencia independencia de campo: influencias culturales e implicaciones para la educación, Colombia, Fondo editorial Universidad Pedagógica Nacional.

Lozano, A. (2001), Estilos de aprendizaje y enseñanza un panorama de la estilística educativa, México, Trillas.

Martin, A (Coord.), (2005), "Identificación de estilos de enseñanza en la universidad. Estudio en tres carreras universitarias: ciencias biomédicas, abogacía y comunicación social", V Coloquio internacional sobre gestión universitaria en América del Sur, Mar de Plata. 
Martin, D. \& Kempa, R. (1991), Los alumnos prefieren diferentes estrategias didácticas en la enseñanza de las ciencias en función de sus características motivacionales, en: Enseñanza de las Ciencias, revista de investigación y experiencias didácticas, núm. 9, pp. 59-68.

Martínez, P. (Abril, 2009), Los estilos de enseñanza conceptualización e investigación: en función de los estilos de enseñanza de Alonso, Galleo y Honey, en: Revista estilos de aprendizaje, núm. 3, vol. 3 .

Mir, P. (2001), Un instrumento de reflexión sobre la actividad docente en el contexto de los programas de formación, disponible en: http://redaberta.usc.es/ aidu/index2.php?option=com_docman\&task=doc view\&gid=106\&Itemid $=8$

Monereo, C. (coord.), (1991), Enseñar a pensar a través del currículo escolar, Barcelona, Casals.

Murillo, J. (2006), Una dirección escolar para el cambio: del liderazgo transformacional al liderazgo distribuido, en: Revista electrónica iberoamericana sobre calidad, eficacia y cambio en educación, vol. 4, núm. 4e.

Traver, J. et al. (2005), Caracterización de las perspectivas docentes del profesorado de secundaria a partir del análisis de las variables educativas relacionadas con la acción y el pensamiento docente, en: Revista Iberoamericana de Educación, núm. 36/8.

Saracho, 0. (2003), Matching teachers and students cognitive styles, en: Early child development and carem, vol.173, Issue 2-3, pp 161-163.

Schön, D. (1992), La formación de profesionales reflexivos. Hacia un nuevo diseño de la enseñanza y el aprendizaje en las profesiones, Madrid, Paidos.

Stenhouse. L. (1985), Investigación y desarrollo del currículo, Madrid, Morata.

Vasquez, A., Martínez, I. (1996), La socialización en la escuela: Una perspectiva Etnográfica, Barcelona, Paidos.

\section{Anexo1.}

\section{Inventario de estilos de enseñanza para docentes universitarios Formato para estudiantes}

El siguiente instrumento tiene como propósito identificar el estilo de enseñanza en los docentes universitarios. Se entienden los estilos de enseñanza como el conjunto de conductas que caracteriza a los docentes durante su accionar en el aula; en ningún caso se busca evaluar al docente identificando las conductas como buenas, malas, deseadas o indeseadas. En esta ocasión se solicita su colaboración como estudiante para identificar las conductas de sus docentes de forma tal que permitan definir sus estilos de enseñanza. Los resultados derivados de este inventario serán usados únicamente con propositivos investigativos y no tendrán incidencia alguna ni en su proceso académico como estudiante, ni en el proceso laboral de su docente.

Por favor complete los siguientes datos de identificación

Género:

Edad: Semestre:

Nombre del docente

Nombre del espacio académico

Por favor lea cuidadosamente cada ítem y marque con una X la casilla correspondiente al nivel de acuerdo con las afirmaciones enunciadas sobre su docente en el espacio académico elegido, teniendo en cuenta las siguientes opciones:

Total Acuerdo (TA)

Moderado Acuerdo (MA)

Moderado Desacuerdo (MD)

Total Desacuerdo (TD) 
cuadro del Anexo 1

\begin{tabular}{|c|c|c|c|c|c|}
\hline & & $\begin{array}{c}\text { Total } \\
\text { Acuerdo }\end{array}$ & $\begin{array}{l}\text { Moderado } \\
\text { Acuerdo }\end{array}$ & $\begin{array}{c}\text { Moderado } \\
\text { Desacuerdo }\end{array}$ & $\begin{array}{c}\text { Total } \\
\text { Desacuerdo }\end{array}$ \\
\hline 1 & $\begin{array}{l}\text { Actúa como mediador cuando existe un conflicto } \\
\text { entre los estudiantes }\end{array}$ & TA & MA & MD & TD \\
\hline 2 & $\begin{array}{l}\text { Los estudiantes pueden acercarse para compartir } \\
\text { sus problemas personales }\end{array}$ & TA & MA & MD & TD \\
\hline 3 & $\begin{array}{l}\text { Identifica el ambiente emocional del grupo tenién- } \\
\text { dolo en cuenta para el desarrollo de la clase }\end{array}$ & TA & MA & MD & TD \\
\hline 4 & $\begin{array}{l}\text { Manifiesta interés por el bienestar de los estudian- } \\
\text { tes más allá del contexto académico }\end{array}$ & TA & MA & MD & TD \\
\hline 5 & $\begin{array}{l}\text { Muestra interés por conocer y vincularse con los } \\
\text { estudiantes }\end{array}$ & TA & MA & MD & TD \\
\hline 6 & Se relaciona con los estudiantes de manera cercana & TA & MA & MD & TD \\
\hline 7 & Reconoce las individualidades dentro del grupo & TA & MA & MD & TD \\
\hline 8 & $\begin{array}{l}\text { Genera un espacio de socialización antes de iniciar } \\
\text { la clase }\end{array}$ & $\mathrm{TA}$ & MA & MD & TD \\
\hline 9 & $\begin{array}{l}\text { Está pendiente de las situaciones personales de } \\
\text { los estudiantes que pueden afectar su desempeño } \\
\text { académico }\end{array}$ & TA & MA & MD & $\mathrm{TD}$ \\
\hline 10 & Saluda a los estudiantes fuera de la clase & TA & MA & MD & TD \\
\hline 11 & $\begin{array}{l}\text { Se preocupa por el progreso individual de los } \\
\text { estudiantes }\end{array}$ & TA & MA & MD & TD \\
\hline 12 & $\begin{array}{l}\text { Es flexible con las actividades propuestas en el } \\
\text { programa }\end{array}$ & TA & MA & MD & TD \\
\hline 13 & Prefiere llegar a acuerdos que imponer decisiones & TA & MA & MD & TD \\
\hline 14 & $\begin{array}{l}\text { En consenso con los estudiantes se plantean las } \\
\text { normas de la clase (hora de ingreso, receso, uso del } \\
\text { celular) }\end{array}$ & TA & MA & MD & TD \\
\hline 15 & $\begin{array}{l}\text { Frente a situaciones imprevistas, el docente acuer- } \\
\text { da con los estudiantes las acciones a seguir }\end{array}$ & TA & MA & MD & TD \\
\hline 16 & $\begin{array}{l}\text { Adapta las temáticas de la clase a los intereses y } \\
\text { necesidades particulares del grupo }\end{array}$ & $\mathrm{TA}$ & MA & MD & TD \\
\hline 17 & $\begin{array}{l}\text { Escucha y toma en cuenta las razones del estudian- } \\
\text { te cuando se equivoca o falla }\end{array}$ & TA & MA & MD & TD \\
\hline 18 & $\begin{array}{l}\text { Introduce la clase enunciando las actividades que } \\
\text { se desarrollaran }\end{array}$ & TA & MA & MD & TD \\
\hline 19 & $\begin{array}{l}\text { Lleva a cabo rutinas claramente establecidas duran- } \\
\text { te la sesión de clase }\end{array}$ & TA & MA & MD & TD \\
\hline 20 & Desarrolla la clase siguiendo una estructura clara & TA & MA & MD & TD \\
\hline 21 & $\begin{array}{l}\text { Retroalimenta el desempeño de los estudiantes a lo } \\
\text { largo del semestre }\end{array}$ & TA & MA & MD & TD \\
\hline 22 & Realiza las evaluaciones planteadas en el programa & TA & MA & MD & TD \\
\hline 23 & Es puntual para comenzar la clase & TA & MA & MD & TD \\
\hline 24 & $\begin{array}{l}\text { Verifica que el estudiante ha realizado una prepara- } \\
\text { ción previa para la clase }\end{array}$ & TA & MA & MD & TD \\
\hline 25 & Es estricto con las fechas de entrega de trabajos & TA & MA & MD & TD \\
\hline 26 & $\begin{array}{l}\text { Exige de sus estudiantes un "comportamiento } \\
\text { adecuado" durante la clase }\end{array}$ & TA & MA & MD & TD \\
\hline 27 & Recuerda constantemente las normas de la clase & TA & MA & MD & TD \\
\hline
\end{tabular}

